\title{
Preoperative serum apolipoprotein A-I levels predict long-term survival in non-muscle-invasive bladder cancer patients
}

This article was published in the following Dove Press journal: Cancer Management and Research

\author{
Zhenhua Shang \\ Jukun Wang \\ Xu Wang \\ Hao Yan \\ Bo Cui \\ Chunsong Jia \\ QiWang \\ Xin Cui \\ Jin $\mathrm{Li}$ \\ Tongwen $\mathrm{Ou}$
}

Department of Urology, Xuanwu Hospital Capital Medical University,

Beijing, People's Republic of China

Correspondence: Tongwen Ou Xuanwu Hospital Capital Medical University, No. 45 Changchun Street, Xuanwu District, Beijing 100069, People's Republic of China

$\mathrm{Tel}+8613501065134$

Email outongwen1967@।26.com
Introduction: The aim of this study was to elucidate the association between apolipoprotein A-I (Apo A-I) and overall survival (OS) as well as cancer-specific survival (CSS) in non-muscleinvasive bladder cancer (NMIBC) patients undergoing transurethral resection of bladder tumor (TURBT).

Patients and methods: We retrospectively collected data of 470 eligible patients diagnosed with NMIBC and who received TURBT between January 2004 and December 2011. Pretreatment blood indexes were examined. The association of Apo A-I with clinicopathological characteristics was further analyzed by dichotomizing our sample into those with Apo A-I $\leq$ $1.19 \mathrm{~g} / \mathrm{L}$ (low Apo A-I group) and those with Apo A-I > $1.19 \mathrm{~g} / \mathrm{L}$ (high Apo A-I group). OS and CSS were estimated by Kaplan-Meier analysis and the log-rank test was used to compare differences between groups. Univariate and multivariate Cox regression analyses were plotted to assess the prognostic value of Apo A-I in NMIBC patients. In addition, subgroup analyses were performed according to the risk classification of the International Bladder Cancer Group. Results: In the overall population, patients in the high Apo A-I group had greater 5-year OS and 5-year CSS rates as compared to those in the low Apo A-I group. Kaplan-Meier survival analysis revealed that higher albumin, Apo A-I, and hemoglobin levels were associated with greater OS and CSS while elevated neutrophil-lymphocyte ratio was associated with worse OS and CSS in the overall and high-risk population rather than low- and intermediate-risk population. Furthermore, Apo A-I was shown to be an independent predictor in the overall population (for OS, hazard ratio [HR], 0.364, 95\% confidence interval [CI], 0.221-0.598, $p<0.001$; for CSS, HR, $0.328,95 \%$ CI, $0.185-0.583, p<0.001$ ) and high-risk patients (for OS, HR, $0.232,95 \%$ CI 0.121-0.443, $p<0.001$; for CSS, HR, 0.269, 95\% CI, 0.133-0.541, $p<0.001$ ). Conclusion: These results suggest that Apo A-I level could potentially serve as a useful prognostic indicator for therapeutic decision making in NMIBC patients.

Keywords: apolipoprotein A-I, NMIBC, TURBT, prognosis, overall survival, cancer-specific survival

\section{Introduction}

In 2012, an estimated 429,800 patients were diagnosed with, and 165,100 patients died from, bladder cancer worldwide. ${ }^{1}$ NMIBC, which is confined to the mucosa or lamina propria, accounts for approximately $80 \%$ of all newly diagnosed bladder cancers. ${ }^{2,3}$ TURBT is a typical first-line treatment for NMIBC patients. However, $70 \%$ of patients may suffer from recurrence after TURBT and 5-year recurrence rates are as high as $80 \% .{ }^{4}$ As a result, many patients undergo TURBT a second time. In addition, prognosis is worse in high-risk NMIBC patients; for example, in those with T1G3, 
5-year disease-specific death rates are as high as $11.3 \%{ }^{5}$ Identifying factors that predict poor oncologic outcomes after TURBT might therefore improve therapeutic decision making for NMIBC patients.

As the major protein constituent of HDL, Apo A-I protein is vital for HDL assembly and plays a major role in its atheroprotective function by facilitating reverse cholesterol transport. ${ }^{6}$ Accumulating evidence has revealed associations between Apo A-I and different types of cancer. Specifically, Apo A-I expression is inversely correlated with the risk of developing breast, lung, colon, and ovarian cancer. ${ }^{7-10}$ The US Food and Drug Administration has also approved the use of Apo A-I as a biomarker for detecting incipient tumors in patients with early-stage ovarian cancer. ${ }^{11,12}$ Additionally, elevated preoperative Apo A-I levels are an independent prognostic marker for longer OS in patients with renal cell, ovarian, colorectal, nasopharyngeal, and ureter urothelial carcinoma. ${ }^{13-17}$ This is consistent with animal studies demonstrating that increased Apo A-I levels suppress tumor growth and metastasis in malignant melanoma, Lewis lung, and ovarian tumor models. ${ }^{18,19}$

NLR is widely used as a predictor of oncologic outcomes in NMIBC patients. In this study, we examined the association between Apo A-I levels and prognosis in NMIBC patients to determine whether preoperative Apo A-I levels might also predict OS and CSS in NMIBC patients undergoing TURBT.

\section{Patients and methods}

\section{Patient selection and data collection}

We retrospectively examined data from 470 patients who were initially diagnosed with NMIBC and subsequently underwent TURBT at the Department of Urology, Xuanwu Hospital Capital Medical University, between January 2004 and December 2011. Patients without previous or coexisting tumors and for whom results of routine blood tests and blood biochemical indexes (including NEUT, LYMPH, PLT, NLR, PLR, HGB, TP, ALB, GLB, A/G, PAB, TG, TC, HDL-C, LDL-C, Apo A-I, and Apo B) prior to treatment were available were included in the study. Patients for whom clinical and pathological information was incomplete, who had non-urothelial bladder carcinoma, or who had other types of concomitant malignant tumors were excluded. Pathological stage was assessed based on the 2010 American Joint Committee on Cancer classification; the 2004 World Health Organization classifications were used to determine tumor grade. In addition, subgroup analyses were performed after categorizing patients based on IBCG risk classification. ${ }^{20,21}$ Low risk was defined as solitary, low-grade (LG) primary Ta tumors, intermediate risk as multiple or recurrent LG tumors, and high risk as $\mathrm{T} 1$ or Tis or high-grade (HG) tumors. NLR was the ratio of NEUT to LYMPH, and PLR was the ratio of PLT to LYMPH. Clinicopathological features included history of smoking; pathological tumor grade; presence of CIS; lymphovascular invasion; and stage, number, and size of tumors; oncologic outcomes examined included tumor recurrence, progression, OS, and CSS. Recurrence was defined as the first pathologically confirmed tumor relapse in the bladder or upper urinary tract, regardless of tumor stage. Progression was defined as an increase in $\mathrm{T}$ category or tumor grade or development of lymph node or distant metastasis. ${ }^{20} \mathrm{~A}$ second TURBT was routinely performed in patients who were diagnosed with $\mathrm{T} 1$ or $\mathrm{HG}$ bladder cancer on initial TURBT. Patients underwent postoperative adjuvant intravesicle instillations of chemotherapy on the basis of tumor characteristics. Demographic data collected at the time of the TURBT procedure at our hospital were also extracted from patients' records. This research was approved by the Ethics Committee of Xuanwu Hospital Capital Medical University. Written informed consent forms were obtained from all enrolled patients for the use of their medical data in this study.

\section{Patient follow-up}

Patients were routinely monitored every 3 months for the first 2 years after TURBT, twice a year for the following 2 years, and annually thereafter. Follow-up investigations consisted of history, physical examination, routine laboratory studies, and cystoscopy. Computed tomography scan was performed every year to assess bladder and upper tract urothelium recurrences.

\section{Statistical analyses}

Statistical analyses were conducted using SPSS statistical software package 22.0 (IBM, Armonk, NY, USA) and MedCalc version 12.5 (MedCalc Software, Ostend, Belgium). Routine blood test results and blood biochemical indexes are presented as medians with ranges. Differences in continuous variables were assessed using unpaired $t$-tests. Categorical variables were analyzed using Pearson's chisquare test. ROC curve analyses were used to calculate appropriate cut-off values for routine blood test results and blood biochemical indexes. Through dichotomized at each possible cut-off point, the optimal cut-off value was selected with the maximal value of Youden index, when 
the maximal sensitivity and specificity were obtained for predicting 5-year OS. In addition, Cox proportional hazard models were applied to these survival variables measured. The end points for this study were 5-year OS and CSS. OS was defined as the time interval (in months) between the date of surgery and date of death for any reason or last follow-up. CSS was measured as the time interval (in months) from the date of surgery to date of death attributed to NMIBC. OS and CSS were estimated by Kaplan-Meier analysis, and the log-rank test was used to assess differences between groups. Multivariate Cox regression analyses were performed to determine independent prognostic value of variables associated with differences in survival. Patients were treated as censored observations if they were alive at the time of last follow-up. Two-sided $p$-values of less than 0.05 were considered statistically significant.

\section{Results \\ Clinicopathological characteristics of 470 NMIBC patients}

Patient clinicopathological characteristics are shown in Table 1. Of the 470 patients included in this study, 354 $(75.32 \%)$ were male and $188(40.00 \%)$ had a history of smoking. The median age at diagnosis was 70 years with a range of 16 to 91 . Most patients ( $n=342,72.77 \%$ ) had LG carcinoma; only one had concomitant CIS. The median follow-up time was 89 months with a range of 10 to 154 . The median Apo A-I level was $1.09 \mathrm{~g} / \mathrm{L}$ (range 0.46-3.27 $\mathrm{g} / \mathrm{L}$ ) and the median NLR level was 2.01 (range 0.45-20). All patients received intravesical chemotherapy and none exhibited lymphovascular invasion. Additionally, 34 (7.23\%) patients received intra-arterial chemotherapy. The 5-year OS and 5-year CSS rates for the overall patient population were $86.81 \%$ and $90.05 \%$, respectively.

\section{Cut-off value selection for serum Apo A-I and other indexes for 5-year OS prediction}

ROC curve analysis is shown Figure 1. As described previously, ${ }^{22}$ the optimal cut-off value of $1.19 \mathrm{~g} / \mathrm{L}$ for Apo A-I level, with an area under the curve of 0.640 (95\% CI, 0.575-0.700; $p=0.0062$ ), was identified based on this analysis. Similarly, $22.28 \mathrm{~kg} / \mathrm{m}^{2}, 1.94 \times 10^{9} / \mathrm{L}, 1.58 \times 10^{9} / \mathrm{L}, 154 \times 10^{9} / \mathrm{L}, 133 \mathrm{~g} / \mathrm{L}$, $1.97 \mathrm{~g} / \mathrm{L}, 127.27 \mathrm{~g} / \mathrm{L}, 59.78 \mathrm{~g} / \mathrm{L}, 41.11 \mathrm{~g} / \mathrm{L}, 20.88 \mathrm{~g} / \mathrm{L}, 1.68$ $\mathrm{mg} / \mathrm{L}, 248 \mathrm{mg} / \mathrm{L}, 1.10 \mathrm{mmol} / \mathrm{L}, 3.18 \mathrm{mmol} / \mathrm{L}, 0.83 \mathrm{mmol} / \mathrm{L}$, $1.69 \mathrm{mmol} / \mathrm{L}$, and $0.86 \mathrm{~g} / \mathrm{L}$ were chosen for BMI, NEUT, LYMPH, PLT, HGB, NLR, PLR, TP, ALB, GLB, A/G, PAB,
TG, TC, HDL-C, LDL-C, Apo B levels, respectively (data not shown). The Apo A-I cut-off value of $1.19 \mathrm{~g} / \mathrm{L}$ was used to divide patients into low $(\leq 1.19 \mathrm{~g} / \mathrm{L})$ and high $(>1.19 \mathrm{~g} / \mathrm{L})$ Apo A-I groups. Accordingly, 326 (69.36\%) patients were assigned to the low Apo A-I group and 144 (30.64\%) to the high Apo A-I group.

\section{Associations between preoperative Apo A-I level and clinicopathological characteristics}

Comparisons of clinicopathological characteristics between the low and high Apo A-I groups are shown in Table 2. Patients with high Apo A-I levels were more likely to have higher ALB $(p=0.033)$, PAB levels $(p=0.003)$, and better 5 -year OS $(p=0.001), 5$-year CSS rates $(p=0.040)$ than those with low Apo A-I levels.

\section{Association between Apo A-I,ALB, NLR, HGB and OS, CSS in the overall population}

We examined whether Apo A-I, ALB, HGB levels, and NLR were associated with OS and CSS using Kaplan-Meier survival analysis. Patients were divided into two low and high groups based on preoperative Apo A-I $(\leq 1.19 \mathrm{~g} / \mathrm{L}$ vs. $>1.19$ $\mathrm{g} / \mathrm{L}), \operatorname{ALB}(\leq 41.11 \mathrm{~g} / \mathrm{L}$ vs. $>41.11 \mathrm{~g} / \mathrm{L})$, and HGB $(\leq 133$ $\mathrm{g} / \mathrm{L}$ vs. $>133 \mathrm{~g} / \mathrm{L})$ levels as well as NLR ( $\leq 1.97$ vs. $>1.97)$. Notably, OS and CSS were longer in patients in the high Apo A-I (>1.19 g/L), ALB (>41.11 g/L), and HGB (>133 g/L) groups. In contrast, elevated NLR ( $>1.97)$ was associated with poorer OS and CSS, as shown in Figure 2.

\section{Significant predictors of OS and CSS identified by univariate and multivariate analyses in the overall population}

Results of univariate and multivariate Cox regression analysis of clinicopathological factors associated with OS and CSS are presented in Tables 3 and 4 . Multivariate analysis revealed that Apo A-I level (HR, 0.364; 95\% CI, 0.221-0.598; $p<$ 0.001), age (HR, 2.388; 95\% CI, 1.439-3.964; $p=0.001$ ), BMI (HR, 0.580; 95\% CI, 0.375-0.897; $p=0.014$ ), tumor grade (HR, $1.678 ; 95 \% \mathrm{CI}, 1.057-2.662 ; p=0.028)$, tumor stage (HR, 1.772; 95\% CI, 1.109-2.831; $p=0.017)$, TP level (HR, 0.373; 95\% CI, 0.172-0.809; $p=0.013)$, ALB level (HR, 0.628; 95\% CI, 0.411-0.962; $p=0.032$ ), A/G (HR, 0.421; 95\% CI, 0.261-0.678; $p<0.001$ ), LDL level (HR, 2.310; 95\% CI, 1.038-5.140; $p=0.040$ ), and HGB level (HR, 0.590; 95\% CI, 0.393-0.885; $p=0.011$ ) were 
Table I Clinicopathological characteristics of 470 NMIBC patients treated by TURBT

\begin{tabular}{|c|c|}
\hline Characteristic & Patients, n (\%) \\
\hline \multicolumn{2}{|l|}{ Gender } \\
\hline Female & II $16(24.68)$ \\
\hline Male & $354(75.32)$ \\
\hline \multicolumn{2}{|l|}{ Age (years) } \\
\hline Mean \pm SD & $67.69 \pm 12.45$ \\
\hline Median (range) & $70(16-91)$ \\
\hline \multicolumn{2}{|l|}{ BMI } \\
\hline Mean \pm SD & $24.75 \pm 3.53$ \\
\hline Median (range) & $24.61(15.23-35.88)$ \\
\hline \multicolumn{2}{|l|}{ Smoking } \\
\hline Yes & I88 (40.00) \\
\hline \multicolumn{2}{|c|}{ Blood cell counts, median (range) } \\
\hline NEUT $\left(\times 10^{9} / \mathrm{L}\right)$ & $3.62(1.23-12)$ \\
\hline LYMPH $\left(\times 10^{9} / L\right)$ & I.8 (0.28-4.07) \\
\hline PLT $\left(\times 10^{9} / L\right)$ & $206(|I-4| 5)$ \\
\hline $\operatorname{HGB}(g / L)$ & $138(79-183)$ \\
\hline \multirow{2}{*}{\multicolumn{2}{|c|}{$\begin{array}{l}\text { Systemic inflammatory response parameters, } \\
\text { median (range) }\end{array}$}} \\
\hline & \\
\hline NLR & $2.01(0.45-20)$ \\
\hline PLR & $118.07(10-691.67)$ \\
\hline \multicolumn{2}{|c|}{ Blood biochemistry, median (range) } \\
\hline $\mathrm{TP}(\mathrm{g} / \mathrm{L})$ & $67.09(50-82.73)$ \\
\hline $\operatorname{ALB}(g / L)$ & $4 \mid .34(3|-5| .7)$ \\
\hline GLB (g/L) & $25.18(15.35-38.64)$ \\
\hline $\mathrm{A} / \mathrm{G}$ & $1.64(0.93-2.7 \mathrm{I})$ \\
\hline $\mathrm{PAB}(\mathrm{mg} / \mathrm{L})$ & $242(75-458)$ \\
\hline $\mathrm{TG}(\mathrm{mmol} / \mathrm{L})$ & $1.22(0.39-7.44)$ \\
\hline $\mathrm{TC}(\mathrm{mmol} / \mathrm{L})$ & $4.24(1.04-7.29)$ \\
\hline $\mathrm{HDL}(\mathrm{mmol} / \mathrm{L})$ & $1.12(0.23-3.14)$ \\
\hline LDL (mmol/L) & $2.26(0.6 I-4.8 I)$ \\
\hline Apo A-I (g/L) & $1.09(0.46-3.27)$ \\
\hline Apo B (g/L) & $0.8 \mathrm{I}(0.0 \mathrm{I}-\mathrm{I} .58)$ \\
\hline \multicolumn{2}{|l|}{ No. of tumors } \\
\hline I & 258 \\
\hline $2-7$ & 122 \\
\hline$\geq 8$ & 90 \\
\hline \multicolumn{2}{|l|}{ Tumor size } \\
\hline$<3 \mathrm{~cm}$ & 424 \\
\hline$\geq 3 \mathrm{~cm}$ & 46 \\
\hline Missing & 0 \\
\hline \multicolumn{2}{|l|}{ Pathologic T stage } \\
\hline $\mathrm{Ta}$ & 232 \\
\hline Tis & 0 \\
\hline $\mathrm{TI}$ & 238 \\
\hline Missing & 0 \\
\hline \multicolumn{2}{|l|}{ Tumor grade } \\
\hline PUNLMP & 10 \\
\hline LG & 342 \\
\hline HG & 118 \\
\hline Missing & 0 \\
\hline \multicolumn{2}{|l|}{ Concomitant CIS } \\
\hline Patients & 1 \\
\hline \multicolumn{2}{|c|}{ IBCG risk classification } \\
\hline Low risk & 100 \\
\hline Intermediate risk & 126 \\
\hline High risk & 244 \\
\hline
\end{tabular}

(Continued)
Table I (Continued)

\begin{tabular}{ll}
\hline Characteristic & Patients, n (\%) \\
\hline $\begin{array}{l}\text { Lymphovascular invasion } \\
\text { Yes }\end{array}$ & 0 \\
$\begin{array}{l}\text { Intravesical chemotherapy } \\
\quad \text { Yes }\end{array}$ & 100 \\
Oncological outcomes & \\
$\quad$ Recurrence (bladder) & $224(47.66)$ \\
Recurrence (upper urinary tract) & $16(3.40)$ \\
Progression & $28(5.96)$ \\
$\quad$ Radical cystectomy & $34(7.23)$ \\
Intra-arterial chemotherapy & $34(7.23)$ \\
Survival & \\
5-year OS & $408 / 470(86.8 I)$ \\
5-year CSS & $380 / 422(90.05)$ \\
Follow-up duration, months & $89(10-154)$ \\
$\quad$ Median (range) & \\
\hline
\end{tabular}

Abbreviations: $A / G$, albumin/globulin ratio; ALB, albumin; Apo A-l, apolipoprotein A-I; Apo B, apolipoprotein B; BMI, body mass index; CIS, carcinoma in situ; CSS, cancer-specific survival; GLB, globulin; HDL, high-density lipoprotein-cholesterol; HG, high grade; HGB, hemoglobin; IBCG, International Bladder Cancer Group; LDL, low-density lipoprotein-cholesterol; LG, low grade; LYMPH, lymphocyte count; NEUT, neutrophil count; NLR, neutrophil-lymphocyte ratio; NMIBC, nonmuscle-invasive bladder cancer; OS, overall survival; PAB, prealbumin; PLR, plateletlymphocyte ratio; PLT, platelet count; PUNLMP, papillary urothelial neoplasm of low malignant potential; SD, standard deviation; TC, total cholesterol; TG, triglyceride; TP, total protein; TURBT, transurethral resection of bladder tumor.

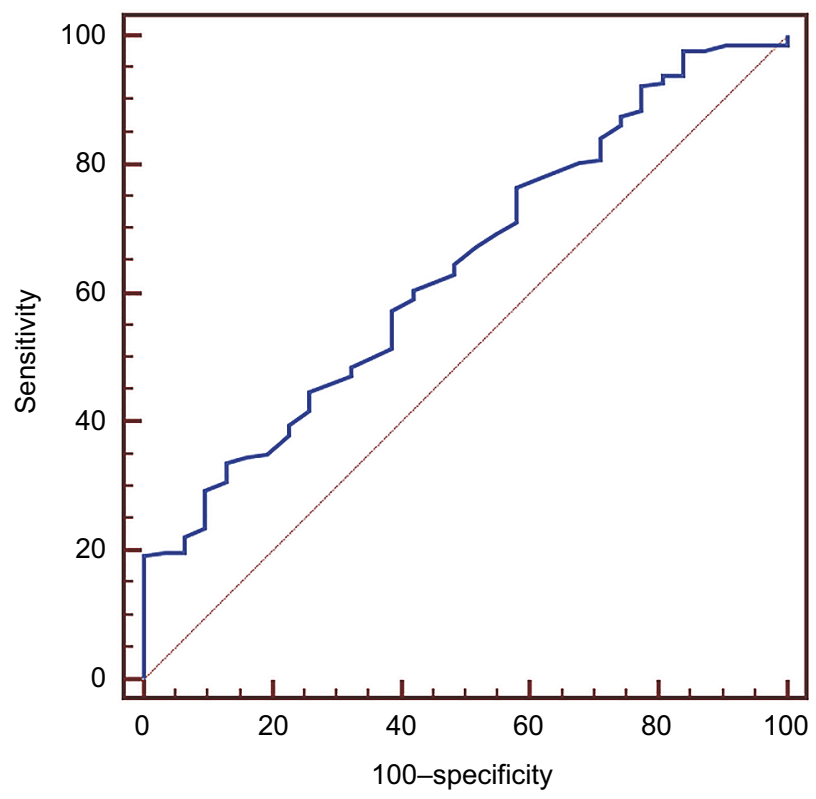

Figure I ROC curve analysis for 5 -year OS prediction. Area under the ROC curve (AUC) was $0.640(95 \% \mathrm{Cl}, 0.575-0.700 ; p=0.0062)$.

Abbreviations: AUC, area under the curve; OS, overall survival; ROC, receiveroperating characteristic.

independent predictive factors for OS after adjusting for other confounding factors. Interestingly, NLR and PLR were of no apparent prognostic significance. Similarly, Apo A-I level (HR, $0.328 ; 95 \%$ CI, $0.185-0.583 ; p<0.001$ ) was also identified as an independent predictor of CSS in the overall patient population. 


\section{Associations between Apo A-I,ALB, NLR, $H G B$ and OS, CSS in subgroup population depending on risk status}

The prognostic significance of Apo A-I, ALB, NLR, and HGB were further assessed in patient subgroups defined by IBCG risk classification. NMIBC patients in each subgroup were further subdivided into two groups according to the cut-off values for Apo A-I, ALB, NLR, and HGB levels. In high-risk patients, as in the overall patient population, elevated Apo A-I (>1.19 g/L), ALB (>41.11 g/L), and HGB levels $(>133 \mathrm{~g} / \mathrm{L})$ were associated with better OS and CSS, while higher NLR $(>1.97)$ was associated with worse OS and CSS (Figure 3). In contrast, low- and intermediate-risk NMIBC patients had similar OS and CSS regardless of high or low Apo A-I, ALB, NLR, and HGB status, which may be due to their high probability of survival (data not shown).

\section{Predictors of OS and CSS in subgroup population depending on risk status}

Factors that predicted OS and CSS were then identified in the IBCG risk classification subgroups. In high-risk patients, age (HR, 2.842; 95\% CI, 1.478-5.467; $p=0.002$ ), BMI (HR, $0.444 ; 95 \% \mathrm{CI}, 0.247-0.799 ; p=0.007$ ), tumor grade (HR, 2.451; 95\% CI, 1.428-4.205; $p=0.001$ ), TP level (HR, 0.247; 95\% CI, 0.116-0.530; $p<0.001$ ), ALB level (HR, 0.486; 95\% CI, 0.274-0.860; $p=0.013$ ), PAB level (HR, 3.404; 95\% CI, 1.812-6.395; $p<0.001$ ), Apo A-I level (HR, 0.232; 95\% CI, 0.121-0.443; $p<0.001$ ), PLT level (HR, 0.472; 95\% CI, 0.223-0.997; $p=0.049$ ), and HGB level (HR, 0.348; 95\% CI, 0.207-0.584; $p<0.001)$ were independent predictive factors for OS (Table 5). Similarly, Apo A-I level (HR, $0.269 ; 95 \% \mathrm{CI}, 0.133-0.541 ; p<0.001)$ was also identified as an independent predictor for CSS in high-risk patients (Table 6). Although univariate analysis revealed that Apo A-I was significantly associated with OS and CSS in the low- and intermediate-risk patients, multivariate analysis indicated that Apo A-I was of no apparent prognostic significance after adjusting for the confounding effects of other variables (data not shown).

\section{Discussion}

To the best of our knowledge, this retrospective study is the first to examine the prognostic value of Apo A-I levels in comparison to other blood indexes, NLR, and PLR in NMIBC patients who underwent TURBT. NLR is an indicator of systemic inflammatory responses and is a well-known predictor of oncologic outcomes in bladder cancer patients. ${ }^{4}$ ALB is also an independent prognostic indicator in many malignancies, such as upper urinary tract urothelial, renal cell, and breast carcinomas. ${ }^{23-25}$ In agreement with these studies, we found here that elevated Apo A-I, ALB, and HGB levels were associated with better OS and CSS, while higher NLR was associated with worse OS and CSS. In addition, univariate analysis revealed that Apo A-I, ALB, and NLR were significantly associated with OS and CSS. However, only Apo A-I was of prognostic significance when all three indicators were included simultaneously in multivariate analysis. We also found that age and tumor grade were the most important prognostic factors for predicting OS and CSS, which was similar to a previous study examining prognostic indicators of OS in NMIBC patients. ${ }^{26}$

Furthermore, analyses of patient subgroups defined by IBCG risk classification showed that elevated Apo A-I, $\mathrm{ALB}$, and HGB levels were associated with better OS and CSS, while higher NLR was associated with worse OS and CSS, in high-risk NMIBC patients. However, in low- and intermediate-risk patients, Apo A-I was not associated with OS or CSS, which may result from their high probability of survival. Apo A-I level was also identified as an independent predictor of OS and CSS in high-risk patients after adjusting for the confounding effects of other variables.

As the major HDL-associated protein, Apo A-I is synthesized primarily in the liver and small intestines and shuttles redundant cholesterol from peripheral organs to the liver for excretion. Apo A-I has been extensively studied as a therapeutic agent for cardiovascular disease. ${ }^{27-29}$ Interestingly, Apo A-I has also been identified as an independent predictor of OS in several cancers. ${ }^{13,15-17,30-32}$ Consistent with these studies, we found here that higher Apo A-I levels were strongly correlated with more favorable OS and CSS in the overall NMIBC patient population and in high-risk patients. Fiveyear OS and CSS rates were $83.44 \%$ and $87.94 \%$, respectively, in patients with Apo A-I levels of $1.19 \mathrm{~g} / \mathrm{L}$ or lower and $94.44 \%$ and $94.29 \%$, respectively, in those with Apo A-I levels higher than $1.19 \mathrm{~g} / \mathrm{L}$. These findings also suggest that Apo A-I might serve as a valuable prognostic indicator in NMIBC patients undergoing TURBT.

Although the mechanisms responsible for the association between Apo A-I and antitumor properties are unclear, several plausible explanations have been proposed. Apo A-I mimetic peptides reduce levels of lysophosphatidic acid, a wellcharacterized promoter of ovarian cancer cell proliferation, in the serum by binding to it with high affinity. ${ }^{19}$ Similarly, treatment of ID8 cells (a mouse epithelial ovarian cancer cell 
Table 2 Characteristics of 470 NMIBC patients grouped by Apo A-I

\begin{tabular}{|c|c|c|c|}
\hline Characteristics & $\begin{array}{l}\text { Apo A-I > } 1.19 \mathrm{~g} / \mathrm{L} \\
(\mathrm{n}=144)\end{array}$ & $\begin{array}{l}\text { Apo A-I } \leq 1.19 \mathrm{~g} / \mathrm{L} \\
(\mathrm{n}=326)\end{array}$ & $p$-value \\
\hline Age (years) & & & 0.690 \\
\hline$>65$ & 90 (62.50\%) & $210(64.42 \%)$ & \\
\hline$\leq 65$ & $54(37.50 \%)$ & 116 (35.58\%) & \\
\hline Gender & & & 0.050 \\
\hline Female & $44(30.56 \%)$ & 72 (22.09\%) & \\
\hline Male & $100(69.44 \%)$ & 254 (77.91\%) & \\
\hline BMI & & & 0.669 \\
\hline$>22.28 \mathrm{~kg} / \mathrm{m}^{2}$ & 110 (76.39\%) & 243 (74.54\%) & \\
\hline$\leq 22.28 \mathrm{~kg} / \mathrm{m}^{2}$ & $34(23.61 \%)$ & $83(25.46 \%)$ & \\
\hline Smoking & & & 0.462 \\
\hline Yes & 54 (37.50\%) & 134 (4I. I0\%) & \\
\hline No & $90(62.50 \%)$ & 192 (58.90\%) & \\
\hline Recurrence & & & 0.480 \\
\hline Yes & 70 (48.61\%) & 170 (52.15\%) & \\
\hline No & 74 (51.39\%) & 156 (47.85\%) & \\
\hline Progression & & & 0.807 \\
\hline Yes & $8(5.56 \%)$ & $20(6.13 \%)$ & \\
\hline No & $136(94.44 \%)$ & 306 (93.87\%) & \\
\hline Tumor grade & & & 0.375 \\
\hline HG & 40 (27.78\%) & 78 (23.93\%) & \\
\hline$\leq \mathrm{LG}$ & $104(72.22 \%)$ & $248(76.07 \%)$ & \\
\hline Tumor stage & & & 0.538 \\
\hline TI & $76(52.78 \%)$ & 162 (49.69\%) & \\
\hline$\leq$ Tis & 68 (47.22\%) & 164 (50.31\%) & \\
\hline Tumor size & & & $0.48 I$ \\
\hline$>3 \mathrm{~cm}$ & 12 (8.33\%) & 34 (10.43\%) & \\
\hline$\leq 3 \mathrm{~cm}$ & I 32 (91.67\%) & $292(89.57 \%)$ & \\
\hline Tumor focality & & & 0.540 \\
\hline Unifocal & 76 (52.78\%) & I 82 (55.83\%) & \\
\hline Multifocal & 68 (47.22\%) & I 44 (44. I7\%) & \\
\hline Radical cystectomy & & & $0.54 I$ \\
\hline Yes & $12(8.33 \%)$ & $22(6.75 \%)$ & \\
\hline No & $132(91.67 \%)$ & 304 (93.25\%) & \\
\hline Intra-arterial chemotherapy & & & $0.54 I$ \\
\hline Yes & 12 (8.33\%) & $22(6.75 \%)$ & \\
\hline No & $132(91.67 \%)$ & 304 (93.25\%) & \\
\hline $\mathrm{TP}$ & & & 0.124 \\
\hline$>59.78 \mathrm{~g} / \mathrm{L}$ & I 35 (93.75\%) & 291 (89.26\%) & \\
\hline$\leq 59.78 \mathrm{~g} / \mathrm{L}$ & $9(6.25 \%)$ & 35 (10.74\%) & \\
\hline ALB & & & 0.033 \\
\hline$>41.11 \mathrm{~g} / \mathrm{L}$ & 86 (59.72\%) & 160 (49.08\%) & \\
\hline$\leq 41.11 \mathrm{~g} / \mathrm{L}$ & $58(40.28 \%)$ & 166 (50.92\%) & \\
\hline GLB & & & 0.084 \\
\hline$>20.88 \mathrm{~g} / \mathrm{L}$ & 134 (93.06\%) & $286(87.73 \%)$ & \\
\hline$\leq 20.88 \mathrm{~g} / \mathrm{L}$ & $10(6.94 \%)$ & 40 (12.27\%) & \\
\hline $\mathrm{A} / \mathrm{G}$ & & & 0.762 \\
\hline$>1.68$ & 64 (44.44\%) & I 40 (42.94\%) & \\
\hline$\leq 1.68$ & $80(55.56 \%)$ & I 86 (57.06\%) & \\
\hline PAB & & & 0.003 \\
\hline$>248 \mathrm{mg} / \mathrm{L}$ & 72 (50.00\%) & II 6 (35.58\%) & \\
\hline$\leq 248 \mathrm{mg} / \mathrm{L}$ & $72(50.00 \%)$ & $210(64.42 \%)$ & \\
\hline TG & & & 0.681 \\
\hline$>1.10 \mathrm{mmol} / \mathrm{L}$ & $8 \mathrm{l}(56.25 \%)$ & 190 (58.28\%) & \\
\hline$\leq 1.10 \mathrm{mmol} / \mathrm{L}$ & 63 (43.75\%) & I 36 (41.72\%) & \\
\hline
\end{tabular}


Table 2 (Continued)

\begin{tabular}{|c|c|c|c|}
\hline Characteristics & $\begin{array}{l}\text { Apo A-I > } 1.19 \mathrm{~g} / \mathrm{L} \\
(\mathrm{n}=144)\end{array}$ & $\begin{array}{l}\text { Apo A-I } \leq 1.19 \mathrm{~g} / \mathrm{L} \\
(\mathrm{n}=326)\end{array}$ & $p$-value \\
\hline TC & & & 0.137 \\
\hline$>3.18 \mathrm{mmol} / \mathrm{L}$ & 104 (72.22\%) & 256 (78.53\%) & \\
\hline$\leq 3.18 \mathrm{mmol} / \mathrm{L}$ & 40 (27.78\%) & 70 (2I.47\%) & \\
\hline $\mathrm{HDL}$ & & & 0.872 \\
\hline$>0.83 \mathrm{mmol} / \mathrm{L}$ & 109 (75.69\%) & 249 (76.38\%) & \\
\hline$\leq 0.83 \mathrm{mmol} / \mathrm{L}$ & 35 (24.31\%) & 77 (23.62\%) & \\
\hline LDL & & & 0.428 \\
\hline$>1.69 \mathrm{mmol} / \mathrm{L}$ & 100 (69.44\%) & 238 (73.0।\%) & \\
\hline$\leq 1.69 \mathrm{mmol} / \mathrm{L}$ & 44 (30.56\%) & 88 (26.99\%) & \\
\hline Apo B & & & 0.205 \\
\hline$>0.86 \mathrm{~g} / \mathrm{L}$ & 52 (36.1 I\%) & I 38 (42.33\%) & \\
\hline$\leq 0.86 \mathrm{~g} / \mathrm{L}$ & 92 (63.89\%) & I 88 (57.67\%) & \\
\hline LYMPH & & & 0.349 \\
\hline$>1.58 \times 10^{9} / \mathrm{L}$ & 94 (65.28\%) & 198 (60.74\%) & \\
\hline$\leq 1.58 \times 10^{9} / \mathrm{L}$ & 50 (34.72\%) & 128 (39.26\%) & \\
\hline NEUT & & & 0.726 \\
\hline$>1.94 \times 10^{9} / \mathrm{L}$ & I 38 (95.83\%) & 310 (95.09\%) & \\
\hline$\leq 1.94 \times 10^{9} / \mathrm{L}$ & $6(4.17 \%)$ & $16(4.91 \%)$ & \\
\hline PLT & & & 0.092 \\
\hline$>154 \times 10^{9} / \mathrm{L}$ & I 28 (88.89\%) & 270 (82.82\%) & \\
\hline$\leq 154 \times 10^{9} / \mathrm{L}$ & 16 (11.11\%) & 56 (17.18\%) & \\
\hline $\mathrm{HGB}$ & & & 0.264 \\
\hline$>133 \mathrm{~g} / \mathrm{L}$ & 84 (58.33\%) & 199 (6I.04\%) & \\
\hline$\leq 133 \mathrm{~g} / \mathrm{L}$ & 60 (4I.67\%) & 127 (38.96\%) & \\
\hline NLR & & & 0.325 \\
\hline$>1.97$ & 68 (47.22\%) & 170 (52.15\%) & \\
\hline$\leq 1.97$ & 76 (52.78\%) & I 56 (47.85\%) & \\
\hline PLR & & & 0.638 \\
\hline$>127.27$ & 62 (43.06\%) & I 48 (45.40\%) & \\
\hline$\leq 127.27$ & 82 (56.94\%) & I 78 (54.60\%) & \\
\hline 5-year OS & I36/I 44 (94.44\%) & $272 / 326(83.44 \%)$ & 0.001 \\
\hline 5-year CSS & I32/140 (94.29\%) & $248 / 282(87.94 \%)$ & 0.040 \\
\hline
\end{tabular}

Note: Bold values indicate statistical significance.

Abbreviations: A/G, albumin/globulin ratio; ALB, albumin; Apo A-I, apolipoprotein A-I; Apo B, apolipoprotein B; BMI, body mass index; CSS, cancer-specific survival; GLB, globulin; HDL, high-density lipoprotein-cholesterol; HG, high grade; HGB, hemoglobin; LDL, low-density lipoprotein-cholesterol; LG, low grade; LYMPH, lymphocyte count; NEUT, neutrophil count; NLR, neutrophil-lymphocyte ratio; NMIBC, non-muscle-invasive bladder cancer; OS, overall survival; PAB, prealbumin; PLR, platelet-lymphocyte ratio; PLT, platelet count; TC, total cholesterol; TG, triglyceride; TP, total protein.

line) with Apo A-I mimetic peptides dramatically reduced cell viability and proliferation by upregulating the antioxidant enzyme MnSOD and decreasing HIF-1 $\alpha$ expression. ${ }^{33,34}$ Apo A-I mimetic peptides can also function as novel antiangiogenesis agents as evidenced by their inhibition of human umbilical vascular endothelial cell proliferation, viability, migration, invasion, and tube formation, ${ }^{35}$ though cancerrelated angiogenesis may be limited in NMIBC patients. Furthermore, Apo A-I inhibits tumor-permissive features of the tumor microenvironment and promotes transformation of tumor-associated macrophages from a pro-tumor M2 to an antitumor M1 phenotype. ${ }^{6,18}$

Moreover, Apo A-I is upregulated in both primary and recurrent bladder cancer patients and may be a potential diagnostic biomarker for bladder cancer. ${ }^{36-39} \mathrm{Li}$ et al found that Apo A-I levels were increased in urine samples from patients with bladder cancer; the authors also noted that the Apo A-I detected in urine from bladder cancer patients was not released from bladder tissue (whether cancerous or morphologically normal). ${ }^{36}$ Investigations into the source of Apo A-I found in urine may improve our understanding of the role Apo A-I plays in bladder cancer. Here, we also found that preoperative Apo A-I levels increased with ALB and PAB levels, but not with NLR, in NMIBC patients. Hypoproteinemia is indicative of systemic inflammatory response, and ALB level is a well-known biomarker for malnutrition. ${ }^{24}$ Low PAB levels also indicate depletion of protein resulting from LG chronic inflammation. ${ }^{40}$ 

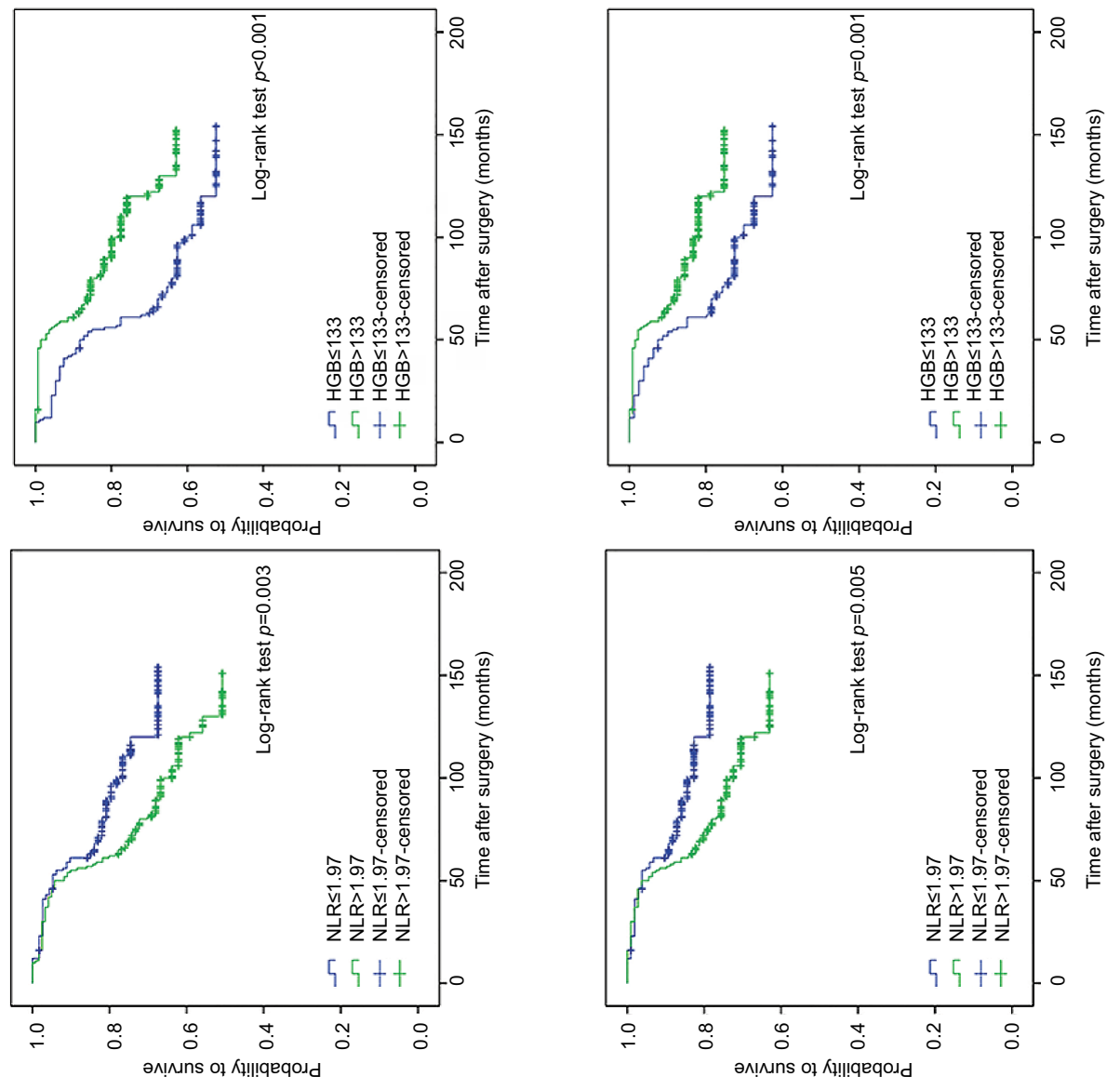

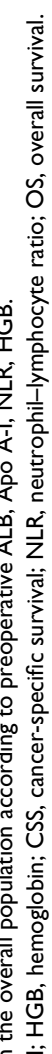
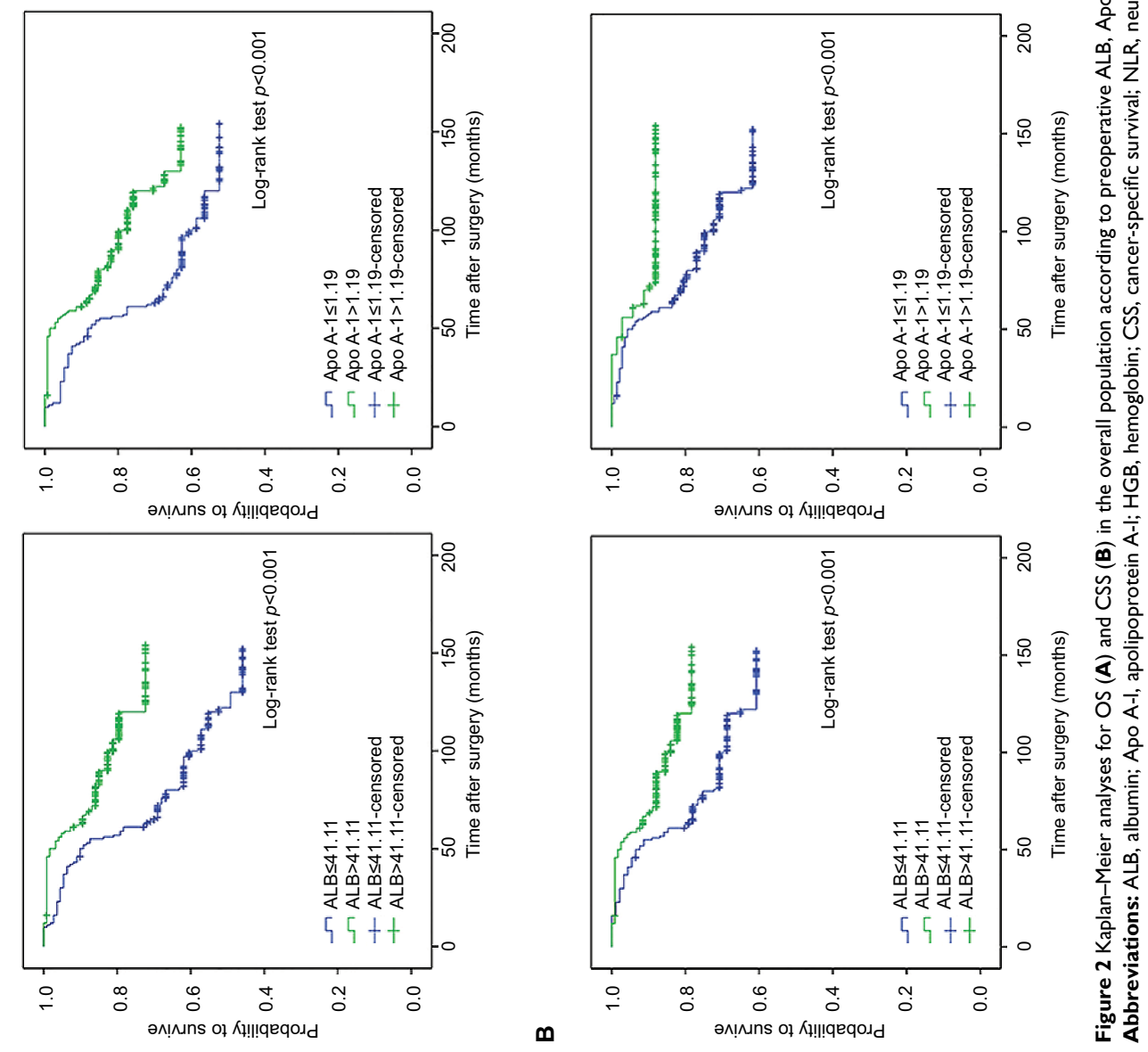
Table 3 Univariate and multivariate analyses to identify the predictive factors for OS in the overall population

\begin{tabular}{|c|c|c|c|c|c|c|}
\hline \multirow[t]{2}{*}{ Characteristic } & \multicolumn{3}{|c|}{ Univariate } & \multicolumn{3}{|c|}{ Multivariate } \\
\hline & $p$-value & HR & $95 \% \mathrm{Cl}$ & $p$-value & HR & $95 \% \mathrm{Cl}$ \\
\hline Age $>65$ years & $<0.001$ & 3.946 & $2.502-6.224$ & 0.001 & 2.388 & I.439-3.964 \\
\hline Female & 0.740 & 0.937 & $0.637-1.377$ & & & \\
\hline $\mathrm{BMI}>22.28 \mathrm{~kg} / \mathrm{m}^{2}$ & 0.002 & $0.58 I$ & $0.410-0.823$ & 0.014 & 0.580 & $0.375-0.897$ \\
\hline Smoking & 0.852 & 1.032 & $0.738-1.443$ & & & \\
\hline Tumor grade & $<0.001$ & 2.384 & $1.70 \mathrm{I}-3.340$ & 0.028 & 1.678 & $1.057-2.662$ \\
\hline Tumor stage & $<0.001$ & 1.962 & $1.390-2.770$ & 0.017 & 1.772 & $\mid .109-2.83 \mathrm{I}$ \\
\hline Tumor size $>3 \mathrm{~cm}$ & 0.853 & 0.952 & $0.564-1.606$ & & & \\
\hline Tumor focality & 0.485 & 1.125 & $0.809-1.563$ & & & \\
\hline Recurrence & 0.009 & $1.57 \mid$ & I.I19-2.204 & 0.090 & 1.420 & $0.947-2.129$ \\
\hline $\mathrm{TP}>59.78 \mathrm{~g} / \mathrm{L}$ & $<0.001$ & 0.257 & $0.168-0.395$ & 0.013 & 0.373 & $0.172-0.809$ \\
\hline ALB $>4 \mathrm{I} .11 \mathrm{~g} / \mathrm{L}$ & $<0.001$ & 0.385 & $0.272-0.545$ & 0.032 & 0.628 & $0.411-0.962$ \\
\hline GLB $>20.88 \mathrm{~g} / \mathrm{L}$ & 0.002 & 0.492 & $0.317-0.764$ & 0.197 & 0.589 & $0.264-1.317$ \\
\hline $\mathrm{A} / \mathrm{G}>\mathrm{I} .68$ & $<0.001$ & 0.452 & $0.314-0.652$ & $<0.001$ & 0.421 & $0.261-0.678$ \\
\hline $\mathrm{PAB}>248 \mathrm{mg} / \mathrm{L}$ & $<0.001$ & 0.443 & $0.309-0.636$ & 0.817 & 1.054 & $0.675-1.647$ \\
\hline $\mathrm{TG}>1.10 \mathrm{mmol} / \mathrm{L}$ & 0.276 & 0.832 & $0.598-1.158$ & & & \\
\hline $\mathrm{TC}>3.18 \mathrm{mmol} / \mathrm{L}$ & 0.003 & 1.936 & $1.248-3.005$ & 0.464 & 1.426 & $0.55 I-3.694$ \\
\hline $\mathrm{HDL}>0.83 \mathrm{mmol} / \mathrm{L}$ & 0.016 & 1.666 & I.098-2.528 & 0.112 & 0.605 & $0.326-1.124$ \\
\hline $\mathrm{LDL}>1.69 \mathrm{mmol} / \mathrm{L}$ & 0.006 & 1.758 & I. $177-2.625$ & 0.040 & 2.310 & $1.038-5.140$ \\
\hline Apo A-I > $1.19 \mathrm{~g} / \mathrm{L}$ & $<0.001$ & 0.323 & $0.201-0.518$ & $<0.001$ & 0.364 & $0.221-0.598$ \\
\hline Apo $B>0.86 \mathrm{~g} / \mathrm{L}$ & 0.827 & 1.038 & $0.745-1.446$ & & & \\
\hline LYMPH $>1.58 \times 10^{9} / \mathrm{L}$ & $<0.001$ & 0.527 & $0.379-0.734$ & 0.952 & 1.015 & $0.620-1.662$ \\
\hline NEUT $>1.94 \times 10^{9} / \mathrm{L}$ & 0.019 & 0.462 & $0.242-0.882$ & 0.968 & 1.017 & $0.447-2.312$ \\
\hline $\mathrm{PLT}>154 \times 10^{9} / \mathrm{L}$ & $<0.001$ & 0.444 & $0.30 \mathrm{I}-0.654$ & 0.456 & 0.815 & $0.476-1.396$ \\
\hline $\mathrm{HGB}>133 \mathrm{~g} / \mathrm{L}$ & $<0.001$ & 0.476 & $0.342-0.662$ & 0.011 & 0.590 & $0.393-0.885$ \\
\hline NLR $>1.97$ & 0.004 & 1.644 & $1.17 \mid-2.307$ & 0.204 & 1.344 & $0.852-2.118$ \\
\hline PLR $>127.27$ & 0.001 & 1.725 & $1.238-2.403$ & 0.286 & 1.276 & $0.816-1.998$ \\
\hline
\end{tabular}

Note: Bold values indicate statistical significance.

Abbreviations: A/G, albumin/globulin ratio; ALB, albumin; Apo A-I, apolipoprotein A-l; Apo B, apolipoprotein B; BMI, body mass index; Cl, confidence interval; GLB, globulin; HDL, high-density lipoprotein-cholesterol; HGB, hemoglobin; HR, hazard ratio; LDL, low-density lipoprotein-cholesterol; LYMPH, lymphocyte count; NEUT, neutrophil count; NLR, neutrophil-lymphocyte ratio; OS, overall survival; PAB, prealbumin; PLR, platelet-lymphocyte ratio; PLT, platelet count; TC, total cholesterol; TG, triglyceride; TP, total protein.

We think the elevated Apo A-I level may result from systemic inflammatory or immune response to bladder tumor, especially through macrophages. It has been found that tumor necrosis factor $\alpha$ could activate endogenous expression and secretion of Apo A-I in human macrophages, which was indeed mediated by mitogen-activated protein kinase cascades..$^{41}$ Moreover, Apo A-I could promote the transformation of tumor-associated macrophages from a pro-tumor M2 to an antitumor M1 phenotype and decrease the percentage of M2 macrophage by preventing their polarization. ${ }^{6,42}$ Interestingly, studies also demonstrated that M2 macrophage infiltration was negatively correlated with better CSS in patients with bladder cancer. ${ }^{43}$ Therefore, we have reasons to believe that macrophages are involved in the association between Apo-I and survival of NMIBC patients. In addition, Apo A-I level was associated with longer survival in patients with renal cell, ovarian, colorectal, nasopharyngeal, and ureter urothelial carcinoma, ${ }^{13-17}$ rather than only in NMIBC patients, which may also indicate a systemic response. Undoubtedly, additional studies are still needed to determine the mechanisms that are responsible for the association between Apo A-I and inflammatory or immune processes in NMIBC patients.

In addition, two issues in this study should be noted: the low rate of concomitant CIS and patients with PUNLMP included. The low rate of concomitant CIS may be attributed to the following reasons. First, some patients may be detected at a later stage and CIS may have progressed to muscle-invasive bladder cancer. For example, studies reported that approximately $54 \%$ of patients with CIS progress to muscle-invasive bladder cancer without treatment. ${ }^{26}$ Second, concomitant CIS was found more in muscle-invasive bladder cancer patients than in NMIBC patients. ${ }^{44}$ Third, the incidence of concomitant CIS was rare naturally and the number of patients was also limited in this study. Regarding the reasons for including patients with PUNLMP, there is no doubt that NMIBC patients comprise those with PUNLMP. Otherwise, a selection bias may result from excluding these patients. Another reason was that PUNLMP was essentially different from benign tumor with 
Table 4 Univariate and multivariate analyses to identify the predictive factors for CSS in the overall population

\begin{tabular}{|c|c|c|c|c|c|c|}
\hline \multirow[t]{2}{*}{ Characteristic } & \multicolumn{3}{|c|}{ Univariate } & \multicolumn{3}{|c|}{ Multivariate } \\
\hline & $p$-value & HR & $95 \% \mathrm{Cl}$ & $p$-value & HR & $95 \% \mathrm{Cl}$ \\
\hline Age $>65$ years & $<0.001$ & 3.266 & $1.952-5.464$ & 0.007 & 2.188 & $1.242-3.852$ \\
\hline Female & 0.339 & 0.802 & $0.510-1.261$ & & & \\
\hline $\mathrm{BMI}>22.28 \mathrm{~kg} / \mathrm{m}^{2}$ & 0.030 & 0.619 & $0.40 \mathrm{I}-0.955$ & 0.140 & 0.677 & $0.403-1.137$ \\
\hline Smoking & 0.713 & 1.080 & $0.717-1.626$ & & & \\
\hline Tumor grade & $<0.001$ & 3.089 & $2.055-4.642$ & 0.010 & 2.039 & $1.190-3.495$ \\
\hline Tumor stage & $<0.001$ & 2.602 & I.672-4.049 & 0.002 & 2.451 & $1.375-4.370$ \\
\hline Tumor size $>3 \mathrm{~cm}$ & 0.905 & 0.961 & $0.497-1.858$ & & & \\
\hline Tumor focality & 0.462 & 1.164 & $0.777-1.745$ & & & \\
\hline Recurrence & 0.014 & 1.680 & I.108-2.547 & 0.120 & 1.485 & $0.902-2.443$ \\
\hline $\mathrm{TP}>59.78 \mathrm{~g} / \mathrm{L}$ & $<0.001$ & 0.313 & $0.177-0.554$ & 0.724 & 0.826 & $0.286-2.386$ \\
\hline$A L B>4 I . I I g / L$ & $<0.001$ & 0.465 & $0.308-0.703$ & 0.080 & 0.632 & $0.378-1.057$ \\
\hline GLB $>20.88 \mathrm{~g} / \mathrm{L}$ & 0.001 & 0.433 & $0.259-0.724$ & $0.04 I$ & 0.384 & $0.153-0.964$ \\
\hline $\mathrm{A} / \mathrm{G}>\mathrm{I} .68$ & 0.005 & 0.541 & $0.353-0.829$ & 0.040 & 0.567 & $0.330-0.975$ \\
\hline $\mathrm{PAB}>248 \mathrm{mg} / \mathrm{L}$ & 0.001 & 0.480 & $0.3|I-0.74|$ & 0.995 & 0.998 & $0.573-1.740$ \\
\hline $\mathrm{TG}>1.10 \mathrm{mmol} / \mathrm{L}$ & 0.758 & 0.938 & $0.623-1.4 I I$ & & & \\
\hline $\mathrm{TC}>3.18 \mathrm{mmol} / \mathrm{L}$ & 0.154 & 1.433 & $0.874-2.350$ & & & \\
\hline $\mathrm{HDL}>0.83 \mathrm{mmol} / \mathrm{L}$ & 0.268 & 1.312 & $0.812-2.121$ & & & \\
\hline $\mathrm{LDL}>1.69 \mathrm{mmol} / \mathrm{L}$ & 0.080 & 1.528 & $0.95 \mathrm{I}-2.456$ & & & \\
\hline Apo A-I > I. $19 \mathrm{~g} / \mathrm{L}$ & $<0.001$ & 0.381 & $0.222-0.653$ & $<0.001$ & 0.328 & $0.185-0.583$ \\
\hline Apo $B>0.86 \mathrm{~g} / \mathrm{L}$ & 0.575 & 1.123 & $0.748-1.687$ & & & \\
\hline LYMPH $>1.58 \times 10^{9} / \mathrm{L}$ & 0.022 & 0.622 & $0.413-0.935$ & 0.342 & 1.340 & $0.733-2.449$ \\
\hline NEUT $>1.94 \times 10^{9} / \mathrm{L}$ & 0.117 & 0.515 & $0.224-1.181$ & & & \\
\hline $\mathrm{PLT}>154 \times 10^{9} / \mathrm{L}$ & $<0.001$ & 0.394 & $0.248-0.627$ & 0.009 & 0.440 & $0.238-0.813$ \\
\hline $\mathrm{HGB}>133 \mathrm{~g} / \mathrm{L}$ & 0.002 & 0.519 & $0.347-0.779$ & 0.024 & 0.581 & $0.362-0.931$ \\
\hline$N L R>1.97^{\circ}$ & 0.006 & 1.802 & I.183-2.745 & 0.035 & 1.795 & $1.042-3.094$ \\
\hline PLR $>127.27$ & 0.049 & 1.502 & $1.002-2.253$ & 0.333 & 1.308 & $0.759-2.253$ \\
\hline
\end{tabular}

Note: Bold values indicate statistical significance.

Abbreviations: A/G, albumin/globulin ratio; ALB, albumin; Apo A-I, apolipoprotein A-I; Apo B, apolipoprotein B; BMI, body mass index; Cl, confidence interval; CSS, cancer-specific survival; GLB, globulin; HDL, high-density lipoprotein-cholesterol; HGB, hemoglobin; HR, hazard ratio; LDL, low-density lipoprotein-cholesterol; LYMPH, lymphocyte count; NEUT, neutrophil count; NLR, neutrophil-lymphocyte ratio; OS, overall survival; PAB, prealbumin; PLR, platelet-lymphocyte ratio; PLT, platelet count; TC, total cholesterol; TG, triglyceride; TP, total protein.

a 35\% recurrence rate. ${ }^{45}$ Moreover, numerous studies that attempted to investigate predictors of oncologic outcomes in NMIBC patients also included PUNLMP patients. ${ }^{4,46,47}$ The retrospective, single-center design of this study, which inherently involves some degree of selection bias, limits the applicability of the results. For example, the optimal cut-off value determined by ROC curve analysis may be different from that calculated by other statistical methods and the limited number of patients may also result in a bias in cut-off value. Prospective, randomized trials are needed to validate our conclusions. In addition, NMIBC patients have relatively long survival times, and follow-up time points beyond 5 years are necessary to confirm these results. Moreover, other combined predictors were not included in our study, which probably should be adjusted as well. Finally, in-depth basic research and clinical studies are needed to identify the mechanisms responsible for the relationship between Apo A-I and survival time in NMIBC patients.

\section{Conclusion}

In this retrospective study of NMIBC patients who underwent TURBT, we found that elevated Apo A-I was associated with better OS and CSS and that higher preoperative serum Apo A-I levels were independent predictors of OS and CSS in the overall patient population and in high-risk patients. These results suggest that Apo A-I might serve as a valuable independent predictor of OS and CSS in NMIBC patients; measurement of Apo A-I levels during routine pretreatment evaluations might therefore improve therapeutic decision making for these patients.

\section{Abbreviations}

$\mathrm{A} / \mathrm{G}$, albumin/globulin ratio

ALB, albumin

Apo A-I, apolipoprotein A-I

Apo B, apolipoprotein B

BMI, body mass index

CI, confidence interval

CIS, carcinoma in situ 

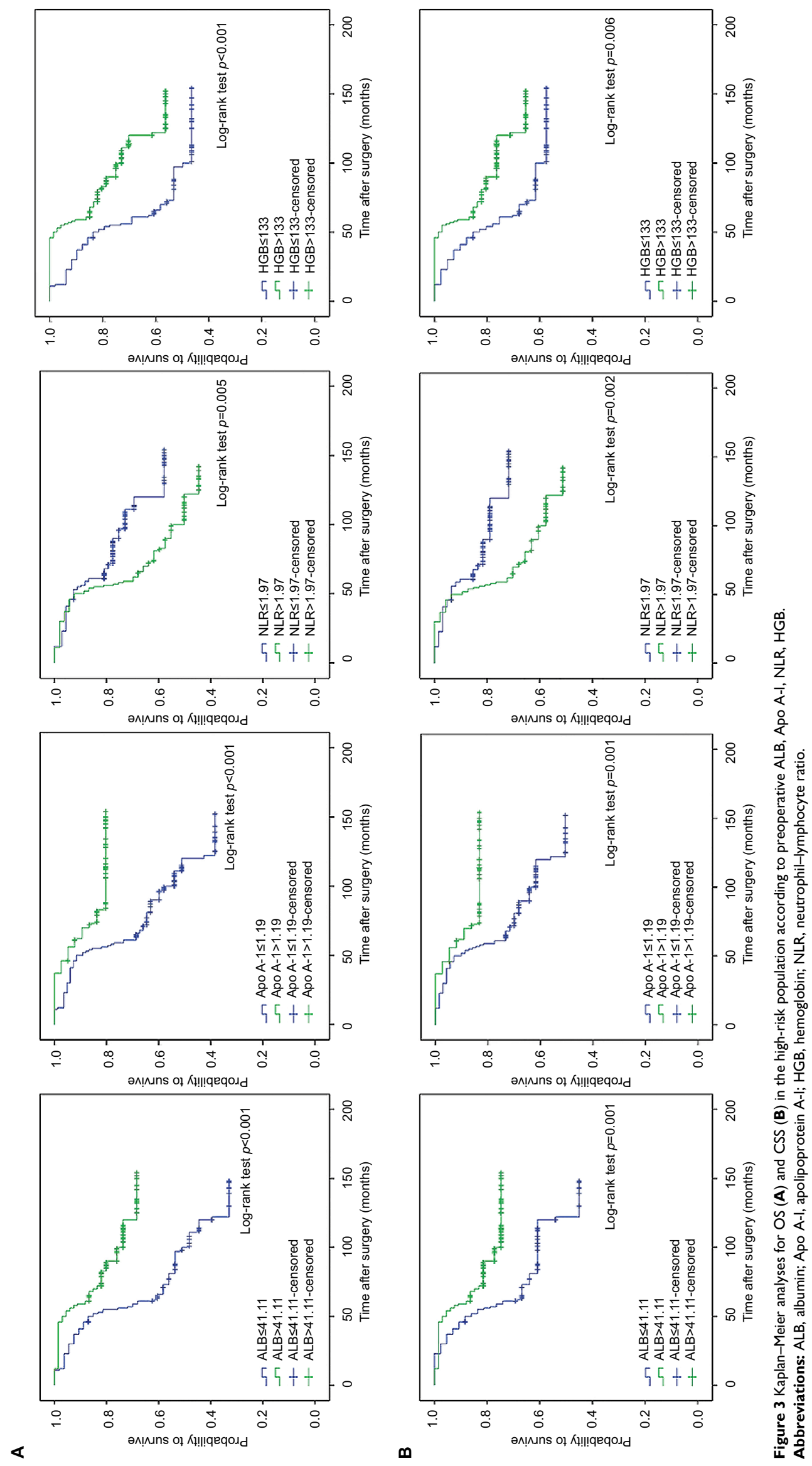
Table 5 Univariate and multivariate analyses to identify the predictive factors for OS in the high-risk population

\begin{tabular}{|c|c|c|c|c|c|c|}
\hline \multirow[t]{2}{*}{ Characteristic } & \multicolumn{3}{|c|}{ Univariate } & \multicolumn{3}{|c|}{ Multivariate } \\
\hline & $p$-value & HR & $95 \% \mathrm{Cl}$ & $p$-value & HR & $95 \% \mathrm{Cl}$ \\
\hline Age $>65$ years & $<0.001$ & 3.846 & $2.170-6.818$ & 0.002 & 2.842 & I.478-5.467 \\
\hline Female & 0.125 & 0.687 & $0.425-1.110$ & & & \\
\hline $\mathrm{BMI}>22.28 \mathrm{~kg} / \mathrm{m}^{2}$ & 0.004 & 0.533 & $0.347-0.820$ & 0.007 & 0.444 & $0.247-0.799$ \\
\hline Smoking & 0.481 & 0.859 & $0.562-1.312$ & & & \\
\hline Tumor grade & 0.001 & 2.036 & $1.338-3.097$ & 0.001 & 2.451 & $1.428-4.205$ \\
\hline Tumor stage & 0.257 & 2.251 & $0.554-9.14 \mid$ & & & \\
\hline Tumor size $>3 \mathrm{~cm}$ & 0.216 & 0.632 & $0.305-1.309$ & & & \\
\hline Tumor focality & 0.701 & 1.086 & $0.7|4-1.65|$ & & & \\
\hline Recurrence & 0.651 & 1.126 & $0.673-1.886$ & & & \\
\hline $\mathrm{TP}>59.78 \mathrm{~g} / \mathrm{L}$ & $<0.001$ & 0.284 & $0.160-0.504$ & $<0.001$ & 0.247 & $0.116-0.530$ \\
\hline ALB $>41.11 \mathrm{~g} / \mathrm{L}$ & $<0.001$ & 0.360 & $0.236-0.550$ & 0.013 & 0.486 & $0.274-0.860$ \\
\hline GLB $>20.88 \mathrm{~g} / \mathrm{L}$ & 0.187 & 0.682 & $0.386-1.205$ & & & \\
\hline $\mathrm{A} / \mathrm{G}>\mathrm{I} .68$ & 0.015 & 0.574 & $0.368-0.896$ & 0.102 & 0.624 & $0.355-1.098$ \\
\hline $\mathrm{PAB}>248 \mathrm{mg} / \mathrm{L}$ & 0.001 & 0.469 & $0.303-0.726$ & $<0.001$ & 3.404 & $1.812-6.395$ \\
\hline $\mathrm{TG}>1.10 \mathrm{mmol} / \mathrm{L}$ & 0.087 & 0.699 & $0.464-1.053$ & & & \\
\hline $\mathrm{TC}>3.18 \mathrm{mmol} / \mathrm{L}$ & 0.006 & 2.174 & $1.243-3.80 \mid$ & $0.77 \mathrm{I}$ & 0.817 & $0.211-3.172$ \\
\hline $\mathrm{HDL}>0.83 \mathrm{mmol} / \mathrm{L}$ & 0.03 I & $1.80 \mathrm{I}$ & $1.055-3.076$ & 0.814 & 1.116 & $0.446-2.796$ \\
\hline $\mathrm{LDL}>1.69 \mathrm{mmol} / \mathrm{L}$ & 0.029 & 1.760 & $1.060-2.922$ & 0.204 & 2.020 & $0.682-5.984$ \\
\hline Apo A-I >I. $19 \mathrm{~g} / \mathrm{L}$ & $<0.001$ & 0.310 & $0.175-0.549$ & $<0.001$ & 0.232 & $0.121-0.443$ \\
\hline Apo B >0.86 g/L & 0.310 & 0.808 & $0.534-1.220$ & & & \\
\hline$L Y M P H>1.58 \times 10^{9} / \mathrm{L}$ & $<0.001$ & 0.371 & $0.246-0.561$ & 0.594 & 1.222 & $0.585-2.552$ \\
\hline NEUT $>1.94 \times 10^{9} / \mathrm{L}$ & $<0.001$ & 0.302 & $0.155-0.587$ & 0.990 & 0.994 & $0.388-2.543$ \\
\hline $\mathrm{PLT}>154 \times 10^{9} / \mathrm{L}$ & $<0.001$ & 0.308 & $0.198-0.480$ & 0.049 & 0.472 & $0.223-0.997$ \\
\hline HGB $>133 \mathrm{~g} / \mathrm{L}$ & $<0.001$ & 0.455 & $0.302-0.686$ & $<0.001$ & 0.348 & $0.207-0.584$ \\
\hline NLR > 1.97 & 0.006 & I.79| & I.185-2.705 & 0.079 & 1.678 & $0.94|-2.99|$ \\
\hline PLR $>127.27$ & 0.004 & 1.826 & $1.213-2.750$ & 0.713 & 0.897 & $0.503-1.599$ \\
\hline
\end{tabular}

Note: Bold values indicate statistical significance.

Abbreviations: A/G, albumin/globulin ratio; ALB, albumin; Apo A-I, apolipoprotein A-I; Apo B, apolipoprotein B; BMI, body mass index; Cl, confidence interval; GLB, globulin; HDL, high-density lipoprotein-cholesterol; HGB, hemoglobin; HR, hazard ratio; LDL, low-density lipoprotein-cholesterol; LYMPH, lymphocyte count; NEUT, neutrophil count; NLR, neutrophil-lymphocyte ratio; OS, overall survival; PAB, prealbumin; PLR, platelet-lymphocyte ratio; PLT, platelet count; TC, total cholesterol; TG, triglyceride; TP, total protein.

CSS, cancer-specific survival

GLB, globulin

HDL-C, high-density lipoprotein-cholesterol

$\mathrm{HG}$, high grade

HGB, hemoglobin

$\mathrm{HR}$, hazard ratio

IBCG, International Bladder Cancer Group

LDL-C, low-density lipoprotein-cholesterol

LG, low grade

LYMPH, lymphocyte count

NEUT, neutrophil count

NLR, neutrophil-lymphocyte ratio

NMIBC, non-muscle-invasive bladder cancer

OS, overall survival

PAB, prealbumin

PLR, platelet-lymphocyte ratio

PLT, platelet count

PUNLMP, papillary urothelial neoplasm of low malignant potential

ROC, receiver-operating characteristic
TC, total cholesterol

TG, triglyceride

TP, total protein

TURBT, transurethral resection of bladder tumor

\section{Acknowledgment}

The authors thank the staff of the medical record office of the Xuanwu Hospital Capital Medical University, who provided patients' records, and all the staff who supported our study.

\section{Disclosure}

The authors report no conflicts of interest in this work.

\section{References}

1. Torre LA, Bray F, Siegel RL, Ferlay J, Lortet-Tieulent J, Jemal A. Global cancer statistics, 2012. CA Cancer J Clin. 2015;65(2):87-108.

2. Tae BS, Kim JK, Kang M, et al. Prognostic value of impaired estimated glomerular filtration rate in intravesical BCG-treated non-muscleinvasive bladder cancer patients. Sci Rep. 2017;7(1):1380.

3. Lopez-Beltran A, Montironi R. Non-invasive urothelial neoplasms: according to the most recent WHO classification. Eur Urol. 2004;46(2): $170-176$. 
Table 6 Univariate and multivariate analyses to identify the predictive factors for CSS in the high-risk population

\begin{tabular}{|c|c|c|c|c|c|c|}
\hline \multirow[t]{2}{*}{ Characteristic } & \multicolumn{3}{|c|}{ Univariate } & \multicolumn{3}{|c|}{ Multivariate } \\
\hline & $p$-value & HR & $95 \% \mathrm{Cl}$ & $p$-value & HR & $95 \% \mathrm{Cl}$ \\
\hline Age $>65$ & $<0.001$ & 3.306 & $1.763-6.197$ & $0.00 \mathrm{I}$ & 3.348 & I.657-6.766 \\
\hline Female & 0.046 & 0.576 & $0.334-0.991$ & 0.019 & 0.462 & $0.242-0.882$ \\
\hline $\mathrm{BMI}>22.28 \mathrm{~kg} / \mathrm{m}^{2}$ & 0.087 & 0.632 & $0.374-1.069$ & & & \\
\hline Smoking & 0.200 & 0.715 & $0.429-1.194$ & & & \\
\hline Tumor grade & 0.001 & 2.392 & I.447-3.954 & 0.026 & 1.949 & $1.082-3.513$ \\
\hline Tumor stage & 0.432 & 1.759 & $0.430-7.185$ & & & \\
\hline Tumor size $>3 \mathrm{~cm}$ & 0.164 & 0.487 & $0.176-1.343$ & & & \\
\hline Tumor focality & 0.807 & 1.064 & $0.649-1.744$ & & & \\
\hline Recurrence & 0.764 & 0.917 & $0.522-1.611$ & & & \\
\hline $\mathrm{TP}>59.78 \mathrm{~g} / \mathrm{L}$ & 0.087 & 0.480 & $0.207-1.113$ & & & \\
\hline ALB $>41.11 \mathrm{~g} / \mathrm{L}$ & 0.001 & 0.451 & $0.277-0.733$ & 0.001 & 0.343 & $0.186-0.633$ \\
\hline GLB $>20.88 \mathrm{~g} / \mathrm{L}$ & 0.358 & 0.729 & $0.372-1.430$ & & & \\
\hline $\mathrm{A} / \mathrm{G}>1.68$ & 0.115 & 0.668 & $0.404-1.104$ & & & \\
\hline $\mathrm{PAB}>248 \mathrm{mg} / \mathrm{L}$ & 0.010 & 0.516 & $0.312-0.853$ & 0.009 & 2.438 & $1.244-4.779$ \\
\hline $\mathrm{TG}>1.10 \mathrm{mmol} / \mathrm{L}$ & 0.198 & 0.728 & $0.449-1.180$ & & & \\
\hline $\mathrm{TC}>3.18 \mathrm{mmol} / \mathrm{L}$ & 0.099 & 1.668 & $0.908-3.065$ & & & \\
\hline $\mathrm{HDL}>0.83 \mathrm{mmol} / \mathrm{L}$ & 0.144 & 1.576 & $0.856-2.903$ & & & \\
\hline $\mathrm{LDL}>1.69 \mathrm{mmol} / \mathrm{L}$ & 0.060 & $1.78 \mid$ & $0.975-3.252$ & & & \\
\hline Apo A-I > $1.19 \mathrm{~g} / \mathrm{L}$ & 0.001 & 0.361 & $0.193-0.676$ & $<0.001$ & 0.269 & $0.133-0.54 \mid$ \\
\hline Apo $B>0.86 \mathrm{~g} / \mathrm{L}$ & 0.206 & 0.727 & $0.444-1.192$ & & & \\
\hline LYMPH $>1.58 \times 10^{9} / \mathrm{L}$ & 0.001 & 0.431 & $0.264-0.704$ & 0.923 & 1.034 & $0.523-2.045$ \\
\hline NEUT $>1.94 \times 10^{9} / \mathrm{L}$ & 0.012 & 0.337 & $0.145-0.784$ & 0.708 & 1.223 & $0.427-3.50 \mathrm{I}$ \\
\hline $\mathrm{PLT}>154 \times 10^{9} / \mathrm{L}$ & $<0.001$ & 0.280 & $0.167-0.470$ & $<0.001$ & 0.257 & $0.124-0.532$ \\
\hline $\mathrm{HGB}>133 \mathrm{~g} / \mathrm{L}$ & 0.007 & 0.514 & $0.317-0.834$ & 0.036 & 0.553 & $0.318-0.961$ \\
\hline NLR $>1.97$ & 0.003 & 2.138 & $1.304-3.505$ & 0.011 & 2.187 & $1.195-4.004$ \\
\hline PLR $>127.27$ & 0.063 & 1.589 & $0.975-2.589$ & & & \\
\hline
\end{tabular}

Note: Bold values indicate statistical significance.

Abbreviations: A/G, albumin/globulin ratio; ALB, albumin; Apo A-I, apolipoprotein A-I; Apo B, apolipoprotein B; BMI, body mass index; Cl, confidence interval; CSS, cancer-specific survival; GLB, globulin; HDL, high-density lipoprotein-cholesterol; HGB, hemoglobin; HR, hazard ratio; LDL, low-density lipoprotein-cholesterol; LYMPH, lymphocyte count; NEUT, neutrophil count; NLR, neutrophil-lymphocyte ratio; OS, overall survival; PAB, prealbumin; PLR, platelet-lymphocyte ratio; PLT, platelet count; TC, total cholesterol; TG, triglyceride; TP, total protein.

4. Kang M, Jeong CW, Kwak C, Kim HH, Ku JH. Preoperative neutrophillymphocyte ratio can significantly predict mortality outcomes in patients with non-muscle invasive bladder cancer undergoing transurethral resection of bladder tumor. Oncotarget. 2017;8(8):12891-12901.

5. Cambier S, Sylvester RJ, Collette L, et al. EORTC nomograms and risk groups for predicting recurrence, progression, and disease-specific and overall survival in non-muscle-invasive stage Ta-T1 urothelial bladder cancer patients treated with 1-3 years of maintenance Bacillus CalmetteGuerin. Eur Urol. 2016;69(1):60-69.

6. Zamanian-Daryoush M, DiDonato JA. Apolipoprotein A-I and cancer. Front Pharmacol. 2015;6:265.

7. Chang SJ, Hou MF, Tsai SM, et al. The association between lipid profiles and breast cancer among Taiwanese women. Clin Chem Lab Med. 2007;45(9):1219-1223.

8. Borgquist S, Butt T, Almgren P, et al. Apolipoproteins, lipids and risk of cancer. Int J Cancer. 2016;138(11):2648-2656.

9. Kim YW, Bae SM, Lim H, Kim YJ, Ahn WS. Development of multiplexed bead-based immunoassays for the detection of early stage ovarian cancer using a combination of serum biomarkers. PLoS One. 2012;7(9):e44960.

10. van Duijnhoven FJ, Bueno-De-Mesquita HB, Calligaro M, et al. Blood lipid and lipoprotein concentrations and colorectal cancer risk in the European Prospective Investigation into Cancer and Nutrition. Gut. 2011;60(8):1094-1102.

11. Clarke CH, Yip C, Badgwell D, et al. Proteomic biomarkers apolipoprotein $\mathrm{A} 1$, truncated transthyretin and connective tissue activating protein III enhance the sensitivity of CA125 for detecting early stage epithelial ovarian cancer. Gynecol Oncol. 2011;122(3):548-553.
12. Nosov V, Su F, Amneus M, et al. Validation of serum biomarkers for detection of early-stage ovarian cancer. Am J Obstet Gynecol. 2009;200(6):639 e631-e635.

13. Tuft Stavnes H, Nymoen DA, Hetland Falkenthal TE, Kaern J, Trope CG, Davidson B. APOA1 mRNA expression in ovarian serous carcinoma effusions is a marker of longer survival. Am J Clin Pathol. 2014;142(1): $51-57$.

14. Quan Q, Huang Y, Chen Q, et al. Impact of serum apolipoprotein A-I on prognosis and bevacizumab efficacy in patients with metastatic colorectal cancer: a propensity score-matched analysis. Transl Oncol. 2017;10(2):288-294.

15. Guo S, He X, Chen Q, et al. The effect of preoperative apolipoprotein A-I on the prognosis of surgical renal cell carcinoma: a retrospective large sample study. Medicine (Baltimore). 2016;95(12):e3147.

16. Jiang R, Yang ZH, Luo DH, et al. Elevated apolipoprotein A-I levels are associated with favorable prognosis in metastatic nasopharyngeal carcinoma. Med Oncol. 2014;31(8):80.

17. Wen WJ, Chen MK, Qin ZK. Serum apolipoprotein A-1 predicts superior prognosis in upper tract urothelial carcinoma after rapid surgery. Chin J Endourol (Electronic Edition). 2016(03):193-197. Chinese [with English abstract].

18. Zamanian-Daryoush M, Lindner D, Tallant TC, et al. The cardioprotective protein apolipoprotein $\mathrm{A} 1$ promotes potent anti-tumorigenic effects. $J$ Biol Chem. 2013;288(29):21237-21252.

19. Su F, Kozak KR, Imaizumi S, et al. Apolipoprotein A-I (apoA-I) and apoA-I mimetic peptides inhibit tumor development in a mouse model of ovarian cancer. Proc Natl Acad Sci U SA. 2010;107(46):19997-20002. 
20. Mano R, Baniel J, Shoshany O, et al. Neutrophil-to-lymphocyte ratio predicts progression and recurrence of non-muscle-invasive bladder cancer. Urol Oncol. 2015;33(2):67.e61-e67.

21. Brausi M, Witjes JA, Lamm D, et al. A review of current guidelines and best practice recommendations for the management of nonmuscle invasive bladder cancer by the International Bladder Cancer Group. J Urol. 2011;186(6):2158-2167.

22. Hajian-Tilaki K. Receiver operating characteristic (ROC) curve analysis for medical diagnostic test evaluation. Caspian J Intern Med. 2013;4(2):627-635.

23. Ko K, Park YH, Lee JW, Ku JH, Kwak C, Kim HH. Influence of nutritional deficiency on prognosis of renal cell carcinoma (RCC). BJU Int. 2013;112(6):775-780.

24. Cui J, Yu M, Zhang N, et al. Prognostic scores based on the preoperative plasma fibrinogen and serum albumin level as a prognostic factor in patients with upper urinary tract urothelial carcinoma. Oncotarget. 2017;8(40):68964-68973.

25. Lis CG, Grutsch JF, Vashi PG, Lammersfeld CA. Is serum albumin an independent predictor of survival in patients with breast cancer? JPEN J Parenter Enteral Nutr. 2003;27(1):10-15.

26. Babjuk M, Bohle A, Burger M, et al. EAU Guidelines on non-muscleinvasive urothelial carcinoma of the bladder: update 2016. Eur Urol. 2017;71(3):447-461.

27. Camont L, Chapman MJ, Kontush A. Biological activities of HDL subpopulations and their relevance to cardiovascular disease. Trends Mol Med. 2011;17(10):594-603.

28. Degoma EM, Rader DJ. Novel HDL-directed pharmacotherapeutic strategies. Nat Rev Cardiol. 2011;8(5):266-277.

29. Libby P, Ridker PM, Hansson GK. Progress and challenges in translating the biology of atherosclerosis. Nature. 2011;473(7347): 317-325.

30. Wang XP, Li XH, Zhang L, et al. High level of serum apolipoprotein A-I is a favorable prognostic factor for overall survival in esophageal squamous cell carcinoma. BMC Cancer. 2016;16:516.

31. Quan Q, Chen Q, Chen P, et al. Decreased apolipoprotein A-I level indicates poor prognosis in extranodal natural killer/T-cell lymphoma, nasal type. Onco Targets Ther. 2016;9:1281-1290.

32. Luo XL, Zhong GZ, Hu LY, et al. Serum apolipoprotein A-I is a novel prognostic indicator for non-metastatic nasopharyngeal carcinoma. Oncotarget. 2015;6(41):44037-44048.

33. Ganapathy E, Su F, Meriwether D, et al. D-4F, an apoA-I mimetic peptide, inhibits proliferation and tumorigenicity of epithelial ovarian cancer cells by upregulating the antioxidant enzyme MnSOD. Int $J$ Cancer. 2012;130(5):1071-1081.

34. Gao F, Chattopadhyay A, Navab M, et al. Apolipoprotein A-I mimetic peptides inhibit expression and activity of hypoxia-inducible factor1alpha in human ovarian cancer cell lines and a mouse ovarian cancer model. J Pharmacol Exp Ther. 2012;342(2):255-262.
35. Gao F, Vasquez SX, Su F, et al. L-5F, an apolipoprotein A-I mimetic, inhibits tumor angiogenesis by suppressing VEGF/basic FGF signaling pathways. Integr Biol (Camb). 2011;3(4):479-489.

36. Li C, Li H, Zhang T, Li J, Liu L, Chang J. Discovery of Apo-Al as a potential bladder cancer biomarker by urine proteomics and analysis. Biochem Biophys Res Commun. 2014;446(4):1047-1052.

37. Bracha S, McNamara M, Hilgart I, et al. A multiplex biomarker approach for the diagnosis of transitional cell carcinoma from canine urine. Anal Biochem. 2014;455:41-47.

38. Lei T, Zhao X, Jin S, Meng Q, Zhou H, Zhang M. Discovery of potential bladder cancer biomarkers by comparative urine proteomics and analysis. Clin Genitourin Cancer. 2013;11(1):56-62.

39. Frantzi M, van Kessel KE, Zwarthoff EC, et al. Development and validation of urine-based peptide biomarker panels for detecting bladder cancer in a multi-center study. Clin Cancer Res. 2016;22(16):4077-4086.

40. Cai W, Kong W, Dong B, et al. Pretreatment serum prealbumin as an independent prognostic indicator in patients with metastatic renal cell carcinoma using tyrosine kinase inhibitors as first-line target therapy. Clin Genitourin Cancer. 2017;15(3):e437-e446.

41. Shavva VS, Mogilenko DA, Nekrasova EV, et al. Tumor necrosis factor alpha stimulates endogenous apolipoprotein A-I expression and secretion by human monocytes and macrophages: role of MAP-kinases, NF-kappaB, and nuclear receptors PPARalpha and LXRs. Mol Cell Biochem. Epub 2018 Feb 13.

42. Peng M, Zhang Q, Cheng Y, et al. Apolipoprotein A-I mimetic peptide $4 \mathrm{~F}$ suppresses tumor-associated macrophages and pancreatic cancer progression. Oncotarget. 2017;8(59):99693-99706.

43. Aljabery F, Olsson H, Gimm O, Jahnson S, Shabo I. M2-macrophage infiltration and macrophage traits of tumor cells in urinary bladder cancer. Urol Oncol. Epub 2017 Dec 26.

44. Karl A, Grimm T, Jokisch F, Gaisa NT, Stief CG. Nichtmuskelinvasives Harnblasenkarzinom: Aktuelles zu Diagnoseverfahren, lokalen Therapieoptionen und zum Update der WHO-Klassifikation 2016. [Non-muscle invasive bladder cancer: Current aspects of diagnostics, local therapy options and the update of the 2016 WHO classification]. Urologe A. 2016;55(9):1247-1258. German [with English abstract].

45. Cheng L, Montironi R, Lopez-Beltran A. TERT promoter mutations occur frequently in urothelial papilloma and papillary urothelial neoplasm of low malignant potential. Eur Urol. 2017;71(3):497-498.

46. Mbeutcha A, Shariat SF, Rieken M, et al. Prognostic significance of markers of systemic inflammatory response in patients with non-muscleinvasive bladder cancer. Urol Oncol. 2016;34(11):483.e417-e483.e424.

47. Sylvester RJ, Oosterlinck W, Holmang S, et al. Systematic review and individual patient data meta-analysis of randomized trials comparing a single immediate instillation of chemotherapy after transurethral resection with transurethral resection alone in patients with stage pTa-pT1 urothelial carcinoma of the bladder: which patients benefit from the instillation? Eur Urol. 2016;69(2):231-244.
Cancer Management and Research

\section{Publish your work in this journal}

Cancer Management and Research is an international, peer-reviewed open access journal focusing on cancer research and the optimal use of preventative and integrated treatment interventions to achieve improved outcomes, enhanced survival and quality of life for the cancer patient. The manuscript management system is completely online and includes

\section{Dovepress}

a very quick and fair peer-review system, which is all easy to use. Visit http://www.dovepress.com/testimonials.php to read real quotes from published authors. 